\title{
Central and peripheral demyelinating polyneuropathy during Nivolumab treatment in patient with advanced non-small cell lung cancer (NSCLC): A case report
} \author{
Francesca Pini ${ }^{1}$ \\ ${ }^{1}$ Medical Oncology San Giovanni hospital, Rome, Italy \\ ${ }^{2}$ Fondazione Policlinico A. Gemelli IRCCS. Università Cattolica del Sacro Cuore, Rome, Italy \\ ${ }^{3}$ Department of Neurology Memorial Sloan Kettering Cancer Center New York \\ ${ }^{4}$ Radiology and Neuroradiology Unit, Fondazione Policlinico Universitario A. Gemelli, IRCCS, Rome, Italy
}

Olga Martelli ${ }^{*}$, Mauro Minelli ${ }^{1}$, Daniela Bernardo ${ }^{2}$, Raffaele Iorio ${ }^{2}$, Bianca D. Santomasso ${ }^{3}$, Tommaso Verdolotti ${ }^{4}$, Mario Sabatelli ${ }^{2}$ and

\begin{abstract}
Immunotherapy with monoclonal antibodies targeting programmed death 1 (PD-1) or programmed death-ligand 1 (PD-L1) such as Nivolumab, Pembrolizumab (anti- PD-1) and Atezolizumab (anti- PDL-1), has substantially changed the management of advanced non-small cell lung cancer (NSCLC). These agents in $10 \%$ of the case may cause immune-related adverse events (irAEs) G3-4 grade such as colitis, rash, thyroiditis, hypophysitis, hepatitis, pneumonia, nephritis. Moreover, few data exist about neurologic immune-related adverse events (neuro irAEs), with potentially life-threatening events derived from clinical trials, single case reports, or small case series, characterized by really aspecific and confounding symptoms, at the onset. As the use of these agents increases, it is important to recognize neuro irAEs early, because they may be serious and potentially fatal and require immediate immunosuppressive therapy. We present a case of a patient with advanced NSCLC who was treated with Nivolumab therapy as second-line treatment. After 12 infusions, he developed severe weakness involving all limbs, respiratory compromise due to central and peripheral nervous system involvement. Nivolumab was discontinued but, despite immunosuppressive therapy, the clinical course worsened, and the patient died 5 months later. This case report is aimed to underline the consciousness of the exsistence of rare neurologic complications derived from the use of immune-checkpoint inhibitors. In the future a multidisciplinary approach is required, to improve and optimize the effect of this essential and powerful therapy.
\end{abstract}

\section{Background}

In recent years, immune checkpoints inhibitors (ICPi) have significantly improved survival outcomes over cytotoxic therapies and are approved for treatment of different advanced and metastatic malignancies.

Toxicity from ICPi occurs, due to both up-regulation of the immune system and interference with self-tolerance.

The most commonly reported IrAEs deriving by the use of IcPi are dermatologic, gastrointestinal (colitis, hepatitis), pulmonary (pneumonitis), and endocrine events. Neurological adverse events are rare, occurring in $<1 \%$ patients treated in clinical trials [1]. The pathogenesis is unclear, but it is thought they may occur when an antitumor immune response damages the nervous system due to a cross reaction directed to an antigen protein expressed by both cancer cells and neurons. Alternatively, it could be secondary to an unmasking autoimmune condition or some other mechanism [2].

Most of the data on neurologic irAE come from case reports of patients with melanoma treated with Ipilimumab or the combination of Ipilimumab plus Nivolumab [3,4]. Neurologic events reported include Guillain-Barré syndrome (GBS), myasthenia gravis, encephalitis, meningitis, peripheral radiculoneuropathy.

Even more limited are the data about neurological adverse events after Nivolumab therapy for the treatment of NSCLC. Central nervous system demyelination has been described as a side effect of Nivolumab therapy in two case reports in patient affected by melanoma $[5,6]$.

Today immunotherapy stage IV NSCLC, of first line in PDL-1 level $>50 \%$ patients, of first line in combination with chemotherapy and second and third line independently from PDL-1 level, in stage III NSCLC after chemo-radio treatment in PDL-1 level $>1 \%$ is indicated. A new indication for small cell lung cancer (SCLC) in first and second line is expected soon.

With expanded indications of ICPi, it has become increasingly important to identify immunologic adverse events which can lead to morbidity and mortality. It is essential to establish criteria for early recognition that would trigger intervention with an adequate immunosuppressive therapy.

\section{Case report}

A 66 year-old, male, performance status (ECOG) 0, former 30 packyear smokers. In anamnesis evidence, from 2012, of atrial fibrillation,

${ }^{\star}$ Correspondence to: Olga Martelli, Medical Oncology San Giovanni hospital, Rome, Italy, E-mail: omartelli@hsangiovanni.roma.it

Received: April 04, 2020; Accepted: April 23, 2020; Published: April 30, 2020 
treated by cardioversion and conduction fiber ablation and in the same year resection of right shoulder melanoma $0,4 \mathrm{~mm}$ sized, stage $\mathrm{I}$.

In 2014 he was diagnosed with lung adenocarcinoma pT2a pN1 cM0 G2, stage IIIA, EGFR wild type, ALK non rearrangement, K-RAS negative. He underwent right superior lobectomy, followed by chemotherapy with cisplatin vinorelbine for four cycles. In 2015 mediastinal lymph node relapse was observed, which was treated with radiotherapy. After that he was affected by pneumonitis and herpes conjuntivis.

In 2016 he was found to have pulmonary progression of disease and was started on second line treatment with Cisplatin and Pemetrexed for four cycles followed by two maintenance Pemetrexed cycles. He stopped in January 2017 due to new pulmonary disease progression. In March 2017 he arrived in our Center where with Nivolumab $3 \mathrm{mg} / \mathrm{kg}$ every two weeks (total dose $360 \mathrm{mg}$, patient weight $120 \mathrm{~kg}$ ) was started.

From summer 2017 after 12 doses, a slight weakness of the left arm was noted. In September he presented moderate symmetric leg weakness which slowly but progressively worsened. At that time neurological examination in another clinical center showed a modest paraparetic gait, no sensitive deficit, brisk reflexes.

In January 2018 he developed an onset of dysarthria and dysphagia. As a consequence Nivolumab therapy was interrupted and prednisone $50 \mathrm{mg} /$ day was started. He underwent radiological assessment with body Positron Emission Tomography- computed tomography (PET/ CT) scan, as well as MRI of the brain and spine, and all resulted negative. Corticosteroid therapy with prednisone $50 \mathrm{mg}$ day was continued without any symptoms improvement. In March 2018 the Patient needed hospitalization in a Neuromuscular Centre for progression of symptoms. Neurological examination revealed moderate weakness ( $\mathrm{F}=3-4 \mathrm{MRC}$ scale) in proximal and distal muscles in all 4 limbs, spastic dysarthria without tongue atrophy. Tendon reflexes were brisk with a slight asymmetry, Chaddock and Hoffmann signs were present bilaterally. He developed a rapid clinical deterioration with severe tetraparesis, dysphagia for liquids and central hypoventilation necessitating Non Invasive Ventilation. EMG showed a motor-sensory peripheral neuropathy with axonal and demyelinating features. Suspecting Nivolumab- induced irAE, prednisone was increased to $100 \mathrm{mg}$ day and treatment with intravenous immunoglobuline was started ( $2 \mathrm{~g} / \mathrm{kg}$ every 4 weeks) but unsuccessfully. Examination of the cerebrospinal fluid (CSF) revealed elevated protein concentration (110 $\mathrm{mg} / \mathrm{dL}$ ), rare cells without atypia. All other parameters were within normal range,

including PCR analyses for neurotropic viruses and cell count was negative, too. Blood cultures and serology tests for virus screening, including CMV, EBV, VZV, HIV, Toxoplasma virus, RSV, Parotid virus, Echovirus and Borrelia were negative. Paraneoplastic antibody panel was negative. A brain MRI was performed in April and, a hyperintensity T2 signal in the pons was observed (differently from the previous negative one performed in January), suggesting an immunomediate brainstem involvement, that looked like a demyelination zone (Figure 1). MRI of the spine was unremarkable. Thoracic CT showed a stable neoplastic disease. Abdomen and encephalic CT were negative for neoplastic disease. The ongoing working diagnosis was of Nivolumab- inducedimmune-related- peripheral poliradiculoneuropathy associated with brainstem encephalitis. The clinical course was characterized by episodic fever $\left(>38^{\circ} \mathrm{C}\right)$ associated to aspergillum pneumonia, severe dyspnoea, headache and emesis that were treated with specific therapy. EMG done in May, showed a total denervation of the tibial, arm biceps, right common extensor of the fingers and right lateral vast muscles, worse than the EMG done in March. The patient became tetraplegic. Follow up brain MRI was immodificate, the hyperintensity T2 signal in the pons was stable; spine MRI was unremarkable; thoracic CT demonstrated a stable neoplastic disease. The patient developed sepsis due to E. Coli urinary sourse and Aspergillus, E. Coli, Proteus mirabilis and Klebsiella pneumonie. His clinical condition deteriorated, he showed a severe anemia caused by episode of rectorragia and melena, heart failure and respiratory failure. He died at the end of July 2018, 4 months after the admission to the hospital. As all the onco markers remained non significant, and chest CT, brain MRI were negative, we conclude that his death was probably due to toxicity.

\section{Discussion}

Neurological adverse events in patients receiving immune checkpoint inhibitors are rare. They occur in $<1 \%$ of patients treated in clinical trials, ranging from mild headache to potentially lifethreatening encephalitis. The post-marketing experience shows an extending number of case reports that describe neurological adverse events. Some reviews of literature show that the major neurological toxicity is described with Ipilimumab or the association IpilimumabNivolumab in the treatment of melanoma and it is worse than antiPD-1 monotherapy $[1,6,7]$. Neurotoxicity most frequently affects the peripheral nervous system and it is characterized by sensorial and motor peripheral neuropathy, Guillain-Barré-like syndrome, myasthenia gravis type syndromes, neuromuscular disorders.

Neurological adverse events with Nivolumab monotherapy are less frequently described and even less frequent are the co-occurence of neuro irAE affecting both the central and peripheral nervous system at the same time.

An on-going study issued by the U.S. Food and Drug Administration proposes to collect, classify and analyse data on all types of neurologic deterioration in patients treated with Nivolumab. Data on this research will be available in 2021 .

One encephalitis case report is described in a patient with nonsmall cell lung cancer treated with Nivolumab ( $3 \mathrm{mg} / \mathrm{kg}$ every 2 weeks) for approximately 7 months achieving a complete anti-tumour response in a clinical trial. It was a grade 5 adverse event which led the patient

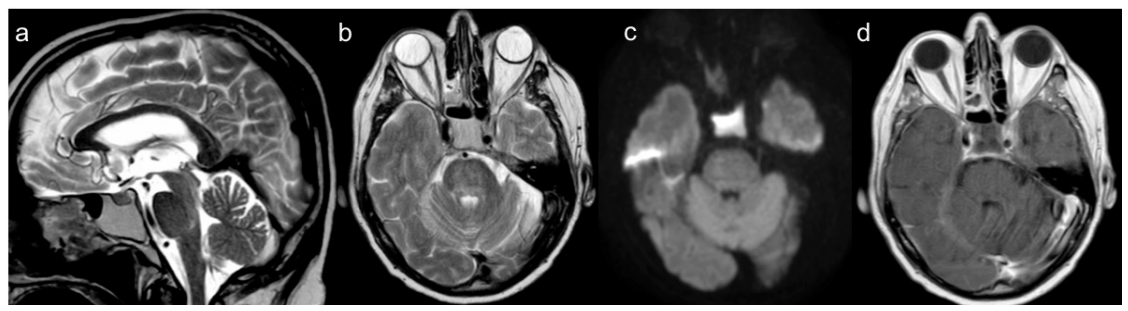

Figure 1. Brain MRI, done in April an hyperintensity T2 signal in the pons appeared, suggesting an immunomediate brainstem involvement, that looked like a demyelination zone 
to death, despite discontinuation of Nivolumab and administration of corticosteroids [8].

A recent review characterize the clinical presentation, diagnostic workup, and management of neuromuscular disorders (NMDs) in patients treated with nivolumab or pembrolizumab monotherapy or concurrent with other immunologic agents, such as ipilimumab. Sixtyone publications on 85 patients. The paper shows that despite adequate treatment strategies including corticosteroid, IV immunoglobulins, and plasma exchange mortality was high in these patients, and that the clinical presentation of NMDs associated with PD-1 inhibitors is often atypical, with considerable overlap between myasthenia gravis and myopathy, and cardiac/respiratory complications are common [9]. Generally, the majority of irAEs can be reversed with discontinuation of the ICPi and corticosteroids therapy, but some patients may need an alternative treatment such as IVIG. Not all patients obtain a complete resolution of neurotoxicity. Some may experience permanent damage or disability or death. Our patient had no response to immunosuppressive therapy and he manifested a slow worsening of neurological symptoms until death for cardio-respiratory failure. At present, very little is known about the pathogenesis of immune-related neurotoxicity, but post mortem pathology analyses have shown lymphocytic inflammation in the involved organs $[4,8]$. Patient's serum was tested for neuralspecific autoantibodies $(\mathrm{Ab})$ by indirect immunofluorescence assays (IFA) on composite substrate of frozen sections of mouse brain, kidney and stomach. IFA revealed an immunoreactivity restricted to the mouse globus pallidus and midbrain (Figure 2). The immunostaining pattern was consistent with an $\mathrm{Ab}$ binding a yet uncharacterized neural antigen. Immunoblot for Ab specific to onconeural antigens and cellbased assays for NMDAR-IgG, Lgi1-IgG, Caspr2-IgG, AMPAR-IgG, GABABR-IgG, mGluR5-IgG, DPPX-IgG, mGluR1-IgG, IgLON5-IgG using commercially available kits (Euroimmun, Luebeck, Germany) yielded negative results. This result supports autoimmune etiology. After discontinuing Nivolumab, advanced NSCLC remained stable, suggesting that the occurrence of irAEs could be linked to its effectiveness. This interesting aspect about the relationship between toxicities and tumour response is still unclear and needs further studies.
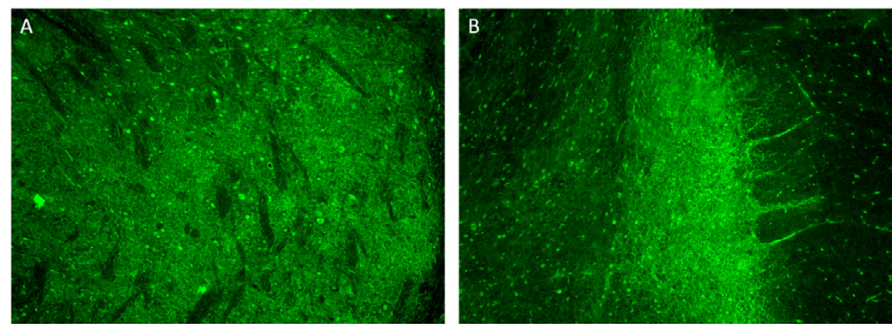

Figure 2. Indirect immunofluorescence assay on mouse brain. IgG in patient's serum (1:200) binds to the midbrain (A) and globus pallidus (B) neuropil; immunoreactivity of the cytoplasm of some neurons can also be observed. Magnification $10 \times$

\section{Conclusion}

Neurological toxicity is rare but can be fatal, and the first symptoms often can be non- specific and slight. Therefore it is important to educate the patients and clinicians about the possibility of immune related neurologic adverse events which can occur such as confusion, seizures, weakness and then progress to severe disability.

Practical guidelines for oncologists are necessary to ensure an early recognition of neurologic symptoms and allow a rapid intervention and an appropriate therapy that leads to their resolution, and it is really important to organize a multidisciplinary team comprising a neurologist in helping to understand and diagnose as soon as possible. Overall evidence suggests that multidisciplinary care facilitates the delivery of a high quality lung cancer service. In this particulary situation a team composed by: oncologist, neurologist, immunologist, dermatologist, endocrinologist, radiotherapist, pneumologist, thoracic surgeon, pathologist, radiologist, etc, is essential to rapidly define the toxicity diagnosis to quickly start the correct care. This could absolutely help the clinician to avoid sending the patients from one specialist to another, often neither updated about immunological therapy and related toxicity. Due to the fundamental importance of a quick and accurate diagnosis, it has resulted essential that patients are kept within the oncology department were they can be followed by the specialized team from the diagnosis to the cure and eventual toxicity.

\section{References}

1. Spain L, Walls G, Julve M, O’Meara K (2016) Neurotoxicity from immune- checkpoint inhibition in the treatment of melanoma: a single centre experience and review of the literature. Ann Oncol 28: 377-385. [Crossref]

2. Hottinger AF (2016) Neurologic complications of immune checkpoint inhibitors. Curr Opin Neurol 29: 806-812. [Crossref]

3. Boutros C, Tarhini A, Routier E, Lambotte O (2016) Safety profiles of anti-CTLA-4 and anti-PD-1 antibodies alone and in combination. Nat Rev Clin Oncol 13: 473-486. [Crossref]

4. Manousakis G, Koch J, Sommerville RB, El-Dokla A, Harms MB, et al. (2013) Multifocal radiculoneuropathy during ipilimumab treatment of melanoma. Muscle Nerve 48: 440-444. [Crossref]

5. Maurice C, Schneider R, Kiehl TR, Bavi P, Roehrl MH, et al. (2015) Subacute CNS Demyelination after Treatment with Nivolumab for Melanoma. Cancer Immunol Res 3: 1299-1302. [Crossref]

6. Larkin J, Chmirlowski B, Lao D, Hodi S, Sharfman W, et al. (2017) Neurologic serious adverse events associated with Nivolumab plus Ipilimumab or nivolumab alone in advanced melanoma, including a case series of encephalities. Oncologist 22: 709-718.

7. Larkin J, Chiarion- Sileni V, Gonzales R (2015) Combined nivolumab and ipilimumab or monotherapy in untreated melanoma. N Engl J Med 373: 1270-1271. [Crossref]

8. Kazandjian D, Suzman DL, Blumenthal G, Mushti S, He K, et al. (2016) FDA Approval Summary: Nivolumab for the treatment of metastatic non small cell lung cancer with progression on or after platinum based chemotherapy. Oncologist 21: 634-42 [Crossref]

9. Johansen A, Christensen SJ, Scheie D, Højgaard JLS, Kondziella D (2019) Neuromuscular advers events associated with anti-PD-1 monoclonal antibodies. Systematic review. Neurology 92: 1-12. [Crossref]

Copyright: (C2020 Martelli O. This is an open-access article distributed under the terms of the Creative Commons Attribution License, which permits unrestricted use, distribution, and reproduction in any medium, provided the original author and source are credited. 